\title{
DISPERSIVE SPECTROSCOPY ON AXAF
}

\author{
T.H. Markert \\ Massachusetts Institute of Technology, Cambridge, MA, USA
}

\begin{abstract}
There are two transmission grating spectrometers and one Bragg crystal spectrometer being developed for the Advanced X-ray Astrophysics Facility (MIT is building the crystal spectrometer and one of the grating spectrometers; the Laboratory for Space Research in Utrecht is responsible for the other grating spectrometer). The gratings divide the AXAF energy band $(80 \mathrm{eV}-10 \mathrm{keV})$ into three regions (the MIT instrument contains gratings with two different periods) and attain resolving powers for point sources between 100 and 1800 . The gratings are composed of arrays of small facets mounted on plates which can be inserted immediately behind the AXAF telescope. The dispersed spectra from the grating arrays are read out by one of the AXAF imaging instruments.
\end{abstract}

The Bragg Crystal Spectrometer (BCS) is a focal plane instrument. One of eight selectable curved diffractors intercepts the AXAF X-ray beam as it diverges beyond the focal point. X-rays that satisfy Bragg's law are reflected from the crystal which, because of its curvature, re-focuses the beam onto an imaging detector. Narrow spectral regions are scanned by rocking the crystal over a range -0.1 to $1^{\circ}$. Nearly the entire AXAF energy range can be studied by selecting the appropriate crystal and rotating it to the proper Bragg angle. The BCS achieves the highest spectral resolutions of the AXAF spectrometers: for $500 \mathrm{eV}<\mathrm{E}<1600 \mathrm{eV}$, the FWHM of a narrow line $(\Delta \mathrm{E})$ is $\leq 1 \mathrm{eV}$.

\section{INTRODUCTION}

Three of the six instruments which are being developed for NASA's Advance Xray Astrophysics Facility (AXAF) are dispersive spectrometers, i.e., the X-radiation is diffracted at a particular angle depending on the wavelength. There are two transmission grating spectrometers and one crystal spectrometer. The Bragg Crystal Spectrometer (BCS) and the High Energy Transmission Grating spectrometer (HETG) are being developed at MIT as part of the High Resolution X-ray Spectroscopy Investigation (Claude Canizares is Principal Investigator). The Laboratory for Space Research in Utrecht, the Netherlands, is designing the Low Energy Transmission Grating spectrometer (LETG). Albert Brinkman is the Principal Investigator.

\section{TRANSMISSION GRATING SPECTROMETERS}

Figure 1 shows, schematically, how the transmission gratings are situated in AXAF (for a detailed description of the HETG and LETG see Canizares, Schattenburg and Smith 1985, Brinkman et al. 1985 and Schattenburg et al. 1988). The gratings are made of small (approximately 1 inch square) elements which are used to cover the slots in a grating assembly plate (there is one plate for the HETG and an independent plate for the LETG). Either grating assembly can be rotated into position immediately behind the 
AXAF telescope so that the slots line up with the telescope annuli (the AXAF mirror assembly consists of 6 Wolter I grazing incidence telescopes; each mirror produces an annulus of $X$-rays with a width of $\leq 2 \mathrm{~cm}$ ). Each grating element (about 500 are required per plate) must be co-aligned to within about 1 arc minute so that the dispersed spectra from each element will superpose correctly.

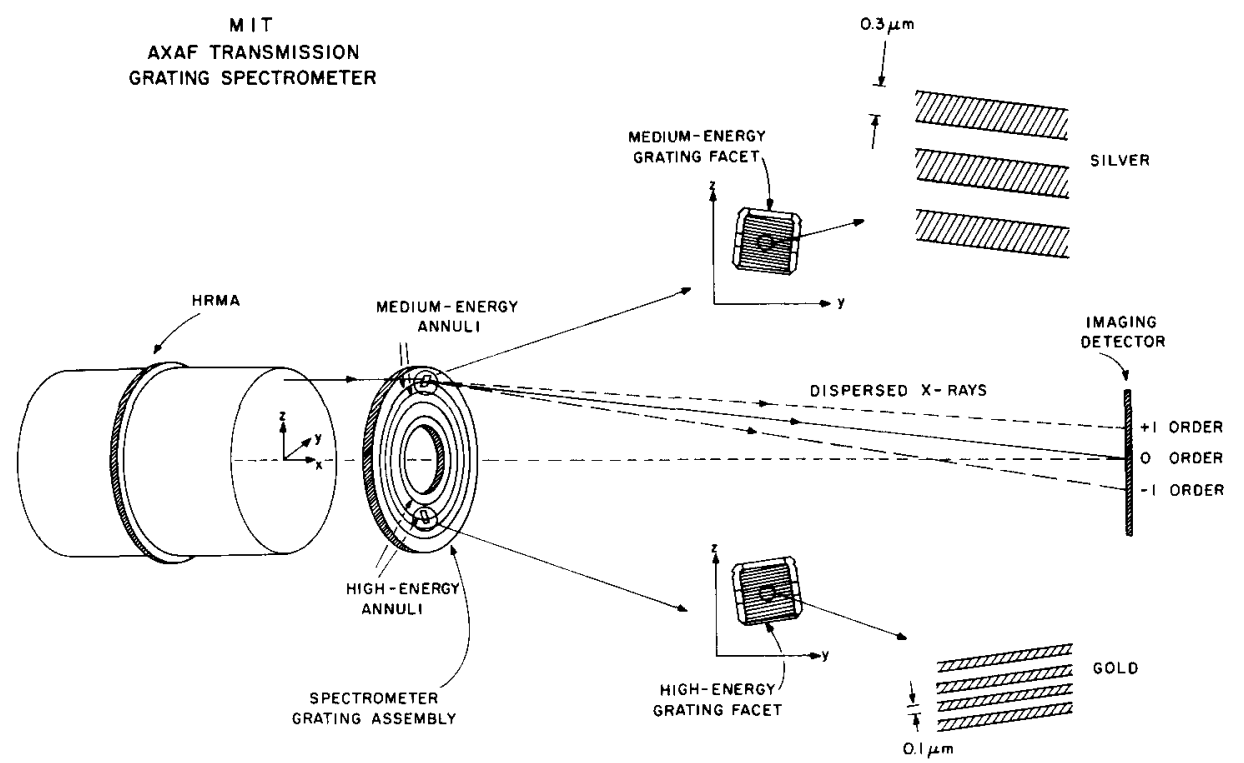

Figure 1 - The MIT Transmission Grating Spectrometer on AXAF (the HETG). 2000 Angstrom period gratings cover the inner three AXAF mirrors (the HEG); 6000 Angstrom period gratings cover the outer three mirrors (the MEG). The Utrecht spectrometer (the LETG) has the same general appearance, except that all of the grating elements are identical (10,000 Angstrom period).

Figure 1 is an illustration of the MIT instrument (the HETG). The HETG is composed of two kinds of gratings elements, $2000 \AA$ period gold gratings (the High Energy Gratings - HEGs) and $6000 \AA$ period silver gratings (the Medium Energy Gratings - the MEGs). The HEGs are situated over the inner three AXAF telescopes (where virtually all of the high-energy response of the AXAF is) and the outer gratings (the MEGs) over the outer three mirrors (where about $70 \%$ of the low-energy response is found). The LETG has a single grating type (10,000 ^ period gold) which covers all 6 of the mirror annuli.

The gratings diffract the X-rays as they emerge from the mirror assembly, dispersing them over the roughly 8.4 meter distance between the grating assembly and the focal plane. The dispersed spectrum is read out by either of the AXAF imaging instruments (the AXAF CCD Imaging Spectrometer [ACIS] or the microchannel plate instrument [the High Resolution Camera, HRC]). The HEG and MEG grating elements (which operate simultaneously as part of the HETG) are tilted slightly with respect to one another. The dispersed spectra from the two grating types form a shallow " $x$ " on the imaging detector, so there is no confusion between the two spectra. 
Figure 2 shows the resolving powers expected for the three grating types. As the figure shows, the three kinds of gratings have high resolution over most of the AXAF energy band. At most energies, $\mathrm{E} / \Delta \mathrm{E}$ is determined by the spatial resolving power (the pixel size) of the imaging instrument and the telescope blur $(E / \Delta E \sim x / \Delta x)$. In order to remove geometric aberrations, the grating assembly and the detector array are designed to approximate a Rowland circle. The residual geometric effects limit the maximum resolving power attainable (e.g. Beuermann, Brauninger and Trumper 1978).

The AXAF gratings have high resolving powers (at most energies) and high sensitivities. Because the resolving power is a function of the image size, the grating spectrometers are most effective for observations of point sources. They can also be used as "slitless spectrometers" to image extended sources in the light of bright lines. Because of the high sensitivity of the transmission gratings, there are a great many candidate sources. For example, we estimate that there are 35,000 active galactic nuclei which can be studied in a reasonable amount of time (i.e. that will provide at least 25 counts in a $200 \mathrm{eV}$ equivalent width line in a 20,000 second observation using the HETG and the ACIS detector).

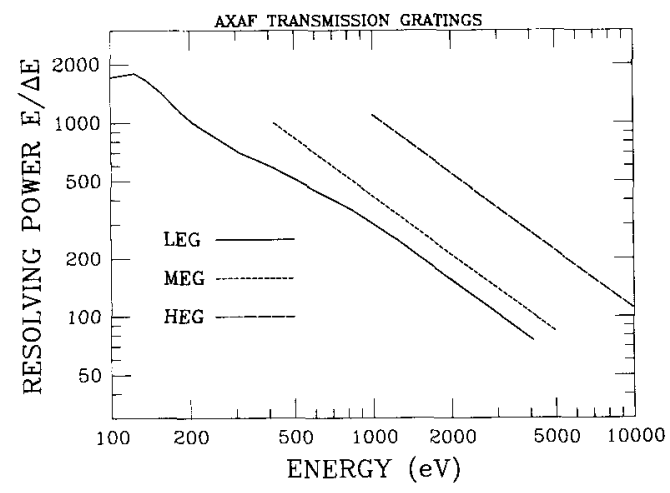

Figure 2 - Resolving powers of the three AXAF Transmission Grating types.

\section{BRAGG CRYSTAL SPECTROMETER}

\subsection{General Description}

The Bragg Crystal Spectrometer (BCS) is an expanded and improved version of the Focal Plane Crystal Spectrometer (FPCS) that operated successfully on the HEAO-2 satellite (for a more detailed discussion of the BCS design, see Canizares, Markert and Clark 1985, and Markert et al. 1988). The BCS is illustrated, schematically, in Figure 3. $\mathrm{X}$-rays from the AXAF telescope come to the focus, where they intercept an assembly containing 10 apertures of different sizes and shapes, a number of filters, and on which sit two sealed monitor proportional counters (for monitoring the time behavior of point sources). If the monitor counters do not intercede, the $\mathrm{X}$-rays continue beyond the focal plane, the beam diverges and strikes one of eight, selectable curved crystals. Those Xrays that satisfy Bragg's law ${ }^{1}$ are reflected efficiently and are refocused onto an imaging proportional counter (there are two for redundancy). The crystal curvature accomplishes two thing: 1) it refocuses the beam so that the X-rays are concentrated onto a very small area (typically $\sim 1 \mathrm{~cm}^{2}$ ). For such a sharp focus the non-X-ray background makes a very small contribution to the signal; 2 ) the diverging $\mathrm{X}$-ray beam strikes the crystal at nearly a

1. $\mathrm{n} \times \lambda=2 \mathrm{~d} \times \sin \theta$, where $\lambda$ is the wavelength, $\mathrm{n}$ is an integer (the order of the diffraction, usually 1$), d$ is the lattice spacing of the crystal, and $\theta$ is the angle between the ray and the tangent to the crystal surface (the Bragg angle) 
constant Bragg angle; therefore the crystal satisfies Bragg's law for only a very narrow range of wavelengths for any one setting of the central angle.

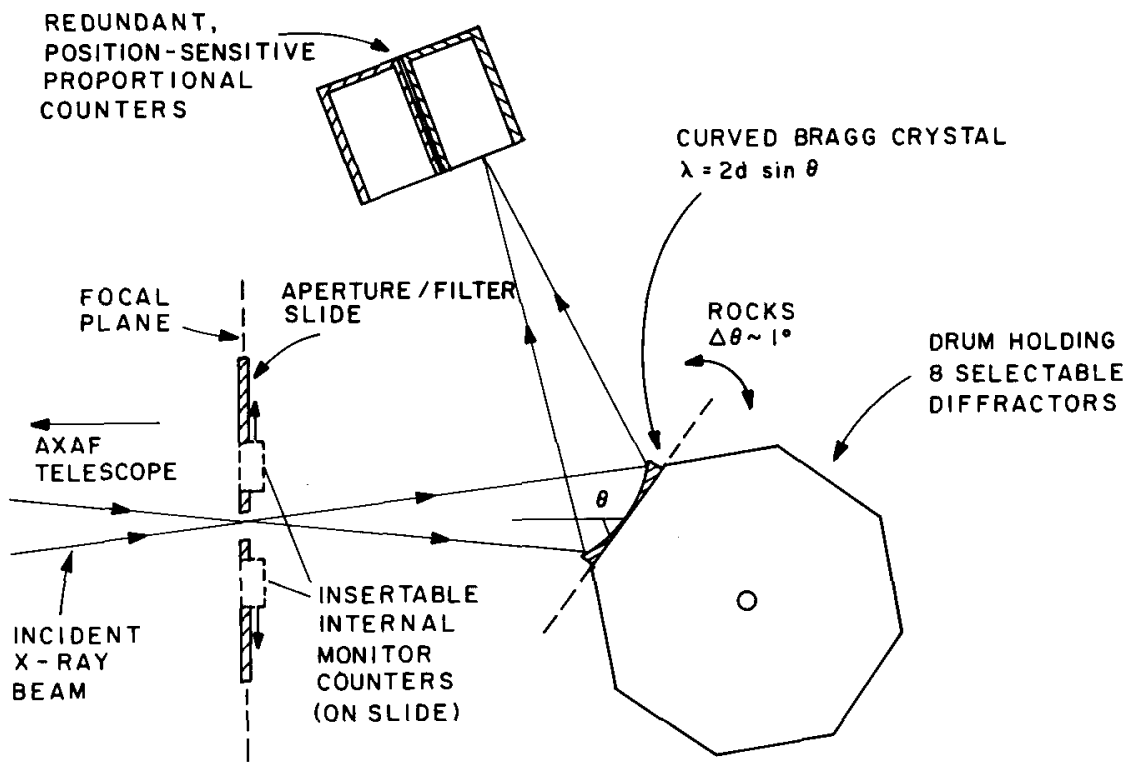

Figure 3 - Schematic of the Bragg Crystal Spectrometer on AXAF.

In order for the crystal curvature to accomplish these two things, it is essential that the three elements of the spectrometer (the X-ray focus, the crystal center and the detector) lie on a circle (a Rowland circle) with diameter equal to the radius of curvature of the crystal itself. This means, for example, that the distance from the crystal to either the detector or the focus must equal $R_{\text {crystal }} \times \sin \theta$. Clearly the crystal must be able to rotate over a large range in order to be able to select a particular wavelength, and the detector must also rotate in order to follow the reflected beam. In total, the Rowland circle geometry requires 5 motor motions. Translating the aperture slide and rotating the drum to select a new crystal add two more motions.

To operate the BCS one selects the appropriate crystal (geometry limits the range of angles accessible to a crystal, so that the wavelength range is also limited; covering the complete AXAF range requires 8 diffractors) and then sets up the Rowland circle for a particular wavelength. The crystal is rotated back and forth over an angle of $\sim 0.1$ to $1.0^{\circ}$. For rocking motions this small, deviations from the ideal Rowland configuration are small. During data reduction, the detector count rate can be correlated with the crystal angle and the spectrum reconstructed.

\subsection{Techniques for Achieving Higher Resolving Power}

As one instrument among 6 on AXAF, the particular strength of the BCS lies in the extremely high spectral resolving powers that it can achieve. One of our goals during 
the AXAF definition phase has been to optimize the BCS to function as a part of a complement of X-ray spectrometers, each of which has certain strengths and weaknesses. Consequently, at MIT we have expended much of our efforts in maximizing the BCS resolving power. By careful selection of diffractor materials and geometries, and by working to conform the crystal shapes to the ideal geometries, we are able to achieve resolving powers significantly better than those of the FPCS (at some energies our goal is $\mathrm{E} / \triangle \mathrm{E}>2000$ ). For example, while all of the FPCS crystals had shapes that were circular in cross-section (so-called Johann geometry) we have baselined a somewhat different shape (the exponential spiral) for two of our crystals. This choice of geometry leads to an extremely high resolving power over a limited range of wavelengths.

We have also been experimenting with another technique for obtaining high resolving power (as suggested, for example, by Schnopper et al. (1976)) which we call the High-Res Setting. This is illustrated in Figure 4. For the standard Johann (diffractor with circular cross-section, Rowland circle configuration) geometry, the diverging $X$ rays strike the crystal at slightly different Bragg angles. In fact, a ray that makes an angle $\alpha$ with the central ray strikes the crystal at a Bragg angle $\theta_{0}+\alpha^{2} / 2 \times \cot \left(\theta_{0}\right)$. Therefore, any one setting of the crystal encompasses a range of wavelengths. This can amount to a broadening of the rocking curve by several arc minutes which, in many cases, can be the dominant factor in limiting the resolution of the crystal. If the detector is moved off the Rowland circle, however, then each X-ray will strike at a different point. Since the detector can image $(\delta x$ $0.5 \mathrm{~mm}$ ), one can trace each ray back to the crystal and thus determine the value of $\alpha$. The Bragg angle can, therefore, be corrected for each ray and the resulting spectrum

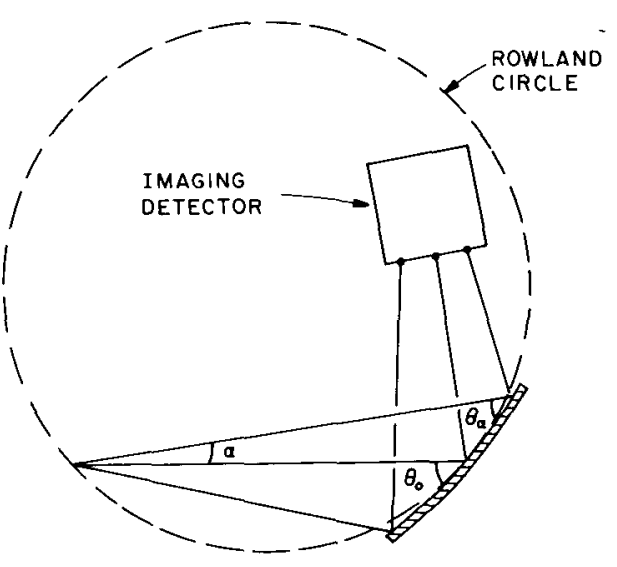

Figure 4 - BCS High-Res Setting. Here the detector is moved off the Rowland circle. The observer uses its imaging capability to determine the source of the ray (the angle $\alpha$ ) and corrects the Bragg angle by $\alpha^{2} / 2 \times \cot \theta_{0}$. made considerably sharper.

We have explored the High-Res setting extensively via computer ray-tracing and in the lab. Figure 5 shows two spectra taken at the MIT crystal evaluation facility. The dashed line is a spectrum of a Ti $\mathrm{K} \alpha$ line taken with the standard setting (i.e, the imaging capabilities of the detector were not used). The solid line is the same data (renormalized) in which the location of the X-ray on the detector was used to correct the Bragg angle (and thus the photon energy). A significant decrease in $\Delta \mathrm{E}$ is seen for the High-Res case.

Figure 6 shows the resolving powers we hope to attain with the BCS in the Standard (detector on the Rowland circle) and the High-Res Settings. Each short line is the resolving power of one of the eight diffractors. The High-Res setting is only useful for observations of point sources, since there is an ambiguity in the image due to location on the sky and location on the crystal. In the Standard Setting, however, the resolving power achieved for observations of extended objects should be as shown in the figure. 
Note that for much of the AXAF energy range, the resolution is better than $1 \mathrm{eV}$ and is $\leqslant$ $10 \mathrm{eV}$ at all energies.

Although achieving high resolving powers is the primary goal of BCS design, we note that, for some kinds of observations, the BCS sensitivity is comparable to that of the other AXAF instruments. In fact, we estimate that there are $-2000 \mathrm{X}$-ray sources in the sky that are suitable targets for detailed spectral analysis with the BCS (i.e., for which we can detect at least 25 photons in at least one X-ray emission line in 20,000 seconds).

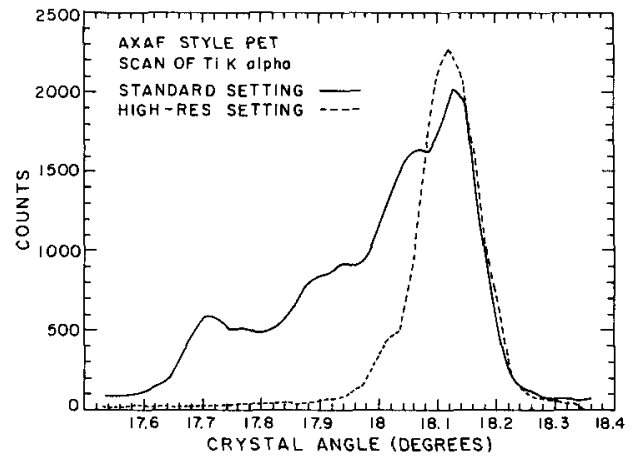

Figure 5 - Spectra of $\mathrm{Ti} \mathrm{K} \alpha$ made with a Bragg Spectrometer at the MIT crystal evaluation facility. Both the Standard (Rowland circle) and the High-Res Settings (detector off the Rowland circle) are shown.

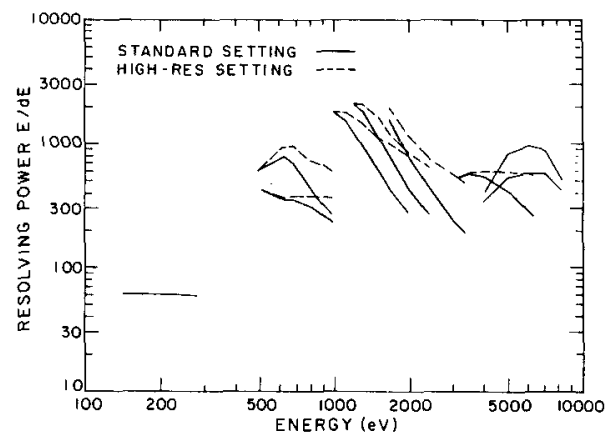

Figure 6 - Resolving powers $(E / \Delta E)$ expected for the BCS on AXAF. Both the Standard Setting (solid lines) and the High-Res Setting (dashed lines) are shown.

ACKNOWLEDGEMENTS. I thank the many workers at MIT and Utrecht who have contributed to the design of the transmission gratings and the BCS. In particular, I am grateful to Mark Schattenburg (HETG Instrument Scientist) for his assistance. This work was supported in part by NASA Contract NAS8-36748.

\section{REFERENCES}

Beuermann, K.P, Brauninger, H., and Trumper, J. 1978, Appl. Optics,17, 2304.

Brinkman, A.C., van Rooijen, J.J., Bleeker, J.A.M., Dijkstra, J.H., Heise, J., de Korte, P.A.J., Mewe, R., and Paerels, F. 1985, Proc. SPIE,597, 232.

Canizares, C.R., Markert, T.H., and Clark, G.W. 1985, Proc. SPIE,597, 241.

Canizares, C.R., Schattenburg, M.L., and Smith, H.I. 1985, Proc. SPIE,597, 253.

Markert, T.H., Powers, T.R., Levine, A.M., McCullum, C.B., Mohr, J.J. and Canizares, C.R. 1988, Proc. SPIE,982, in press.

Schattenburg, M.L., Canizares, C.R., Dewey, D., Levine, A.M., Markert, T.H. and Smith, H.I. 1988, Proc. SPIE, 982, in press.

Schnopper, H.W., Delvaille, J.P., Epstein, A., Kalata, K. and Sohval, R. 1976, Space Science Instrumentation, 2,243. 


\section{DISCUSSION-T. Markert}

J. Vallerga: Do you have any concerns about the stability of the silver transmission gratings on the ground and in orbit?

T. Markert: We are aware of a potential problem with oxygen contamination. On the ground, silver can tarnish (oxidize). We will test tarnished gratings on the ground to see if oxidation affects their performance. In space, oxygen atoms bombard satellites at high velocities causing damage to plastics and silver. This should not be a problem for us except when the telescope is pointing in the direction of orbital motion. If necessary we may deposit a thin coating of non-reactive material on the gratings (e.g. aluminum) or make the gratings out of a non-reactive metal such as ruthenium or rhodium.

J. Linsky: Your high resolution mode produces a narrow, symmetric rocking curve compared to the asymmetric rocking curve for the standard mode. Could you comment on whether or not the high resolution mode also produces more symmetric line profiles.

T. Markert: The High-Resolution Setting removes the systematic geometric effects on the rocking curve shape. The remaining contributors to the rocking curve shape are departures from the nominal geometry that occur during the bending process and the inherent shape of the curve which is a characteristic of the particular crystal. These contributors are reasonably symmetric, particularly for an imperfect crystal, where the mosaic orientations are random. Therefore we expect the profile of a line scanned in the High-Res Setting to be fairly symmetric.

H. Schnopper: When the instrument is in the high-resolution setting what is the penalty in sensitivity which is caused by the larger area on the detector that is required to detect the line?

C. Canizares: Because the background rate of the BCS is so low, most observations are still signal-limited. For very weak sources the increased background makes a difference (it increases by approximately a factor of 10), but only a few objects in the BCS target population are affected by the higher background.

S. Labov: What are the advantages of a Bragg crystal spectrometer if an X-ray calorimeter is built which achieves $1 \mathrm{eV}$ resolution?

T. Markert: A $1 \mathrm{eV}$ X-ray calorimeter would be a formidable detector. The BCS would have some advantages, such as a longer lifetime (the gas supply to the proportional counter would last longer than the liquid helium cryogen), a larger field of view, and a somewhat higher resolution at some energies (we hope to achieve $0.5 \mathrm{eV}$ with the BCS). I think it is important to point out, however, that the best resolution thus far demonstrated with a calorimeter is $17 \mathrm{eV}$ (see Holt's paper in this volume), so that the technology has to advance significantly before crystal spectrometers become obsolete. At the same time that calorimeter technology is being improved, of course, we (and others) will be striving to improve crystal techniques. 